\title{
Self-Induced Convection at Microelectrodes via Electroosmosis and Its Influence on Impact Electrochemistry
}

\author{
Taghi Moazzenzade, Xiaojun Yang, Luc Walterbos, Jurriaan Huskens, Christophe Renault, \\ and Serge G. Lemay*
}

Cite This: J. Am. Chem. Soc. 2020, 142, 17908-17912

Read Online

ACCESS | Llll Metrics \& More | 国 Article Recommendations ｜（s Supporting Information

ABSTRACT: Faradaic reactions at low supporting electrolyte concentrations induce convection via electroosmotic flows. Here we combine finite-element simulations and electrochemical measurements on microparticles at ultramicroelectrodes to explore this effect. We show that convection becomes the dominant form of mass transport for experiments at low salt concentrations, violating the common assumption that convection can be neglected.

$\mathrm{E}$ lectrochemical processes and associated mass transport play a role in a plethora of physical phenomena and applications. While mass transport can often be understood in terms of diffusion alone, ${ }^{1}$ migration and the corresponding ohmic potential drops also become increasingly relevant at low supporting electrolyte ratios. $^{2-7}$ Convection can also be imposed, for example in flow cells or at rotating disk electrodes. Moreover, natural convection has been shown to occur in temperature or concentration gradients and when caused by electrochemically generated bubbles. ${ }^{8}$

Far less recognized, however, is that electrochemical reactions can also induce convection through the generation of electroosmotic flows (EOFs). In this phenomenon, the electrochemically generated electric field drives the migration of mobile ions in the electrical double layer (EDL) of surfaces in contact with the solution, which in turn causes advection of the solvent. Electroosmosis has been intentionally employed in electrochemistry, for example for self-propulsion of microscale swimmers, ${ }^{9}$ microscale pumping, ${ }^{10,11}$ enhancing mass transport in nanoband electrodes, ${ }^{12}$ and directing the motion of bacteria. ${ }^{13}$ The effect is however general and in principle occurs to some degree in most electrochemical processes. The insulating materials found in electrochemical cells consequently play an active role in determining the electrochemical response.

Here we illustrate this behavior experimentally by tracking the motion of microparticles in the vicinity of an ultramicroelectrode (UME) and relating these observations to amperometric signals caused by the oxidation of a redox mediator. ${ }^{14}$ While it is well understood that the frequency of current blockade events increases with decreasing salt concentration, ${ }^{14-16}$ EOFs cause the nature of the amperometric signals to change when the supporting electrolyte ratio ( $\gamma$, defined as the monovalent salt concentration divided by the redox meditator concentration) becomes lower than unity. Convection in fact often dominates particle transport at low ionic strengths.

Miniaturized electrodes such as UMEs are particularly well suited to low-salt conditions because their solution resistance is largely determined by the region near the electrode. ${ }^{3}$ When $\gamma<$ 1 , the ionic strength in this region increases, leading to decreased solution resistance. ${ }^{3}$ However, UMEs are usually shrouded by an insulating surface such as glass which is normally charged in solution. This charge can interact with the induced electric field to cause EOFs and thus locally induce convection.

To gain a quantitative understanding of this effect, we performed finite-element calculations of mass transport including self-generated electric fields and convection. In short, the species were a redox mediator in the reduced form (neutral, $z=0)$, its oxidized counterpart $(z=+1)$, and a supporting electrolyte represented by a cation $(z=+1)$ and an anion $(z=-1)$. Transport was described by the NernstPlanck equation. Charge neutrality was imposed, and consequently, surface charges and the corresponding EDLs were not explicitly included in the equations. It was assumed that the mediator was instantly converted to the oxidized form at the electrode (high overpotential conditions ${ }^{2}$ ). The solution was further described as an incompressible Newtonian fluid. To describe EOFs, the velocity of the fluid at the glass surface, $u_{\|}$, was given by the Helmholtz-Smoluchowski equation, $u_{\|}=$ $\left(\varepsilon \zeta_{s} / \mu\right) E_{\| \cdot}{ }^{17}$ Here $\varepsilon$ and $\mu$ are the permittivity and the viscosity of the solution, respectively, $\zeta_{s}$ is the $\zeta$-potential of the surface, and $E_{\|}$is the tangential electric field. The microparticle trajectories were computed from force balance between electrophoretic and viscous drag forces (see Supporting Information for a complete description).

The self-consistent results are summarized in Figure 1. Oxidation of the mediator at the electrode causes local injection of positive charge into the solution and gives rise to

Received: August 6, 2020

Published: October 12, 2020 

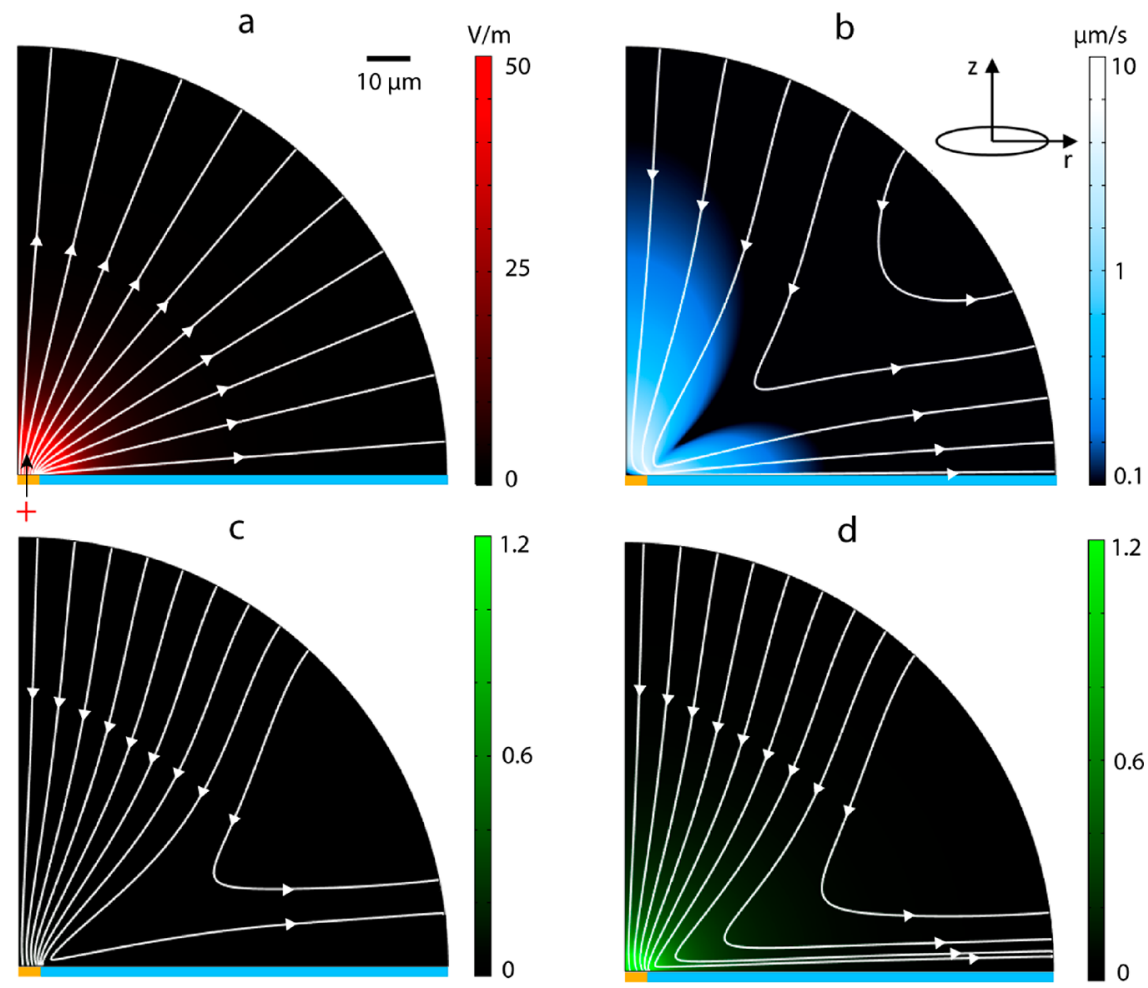

Figure 1. Calculated mass transport properties. (a) Electric field lines (white) and magnitude (color scale) upon oxidation at the electrode. This field causes a $1.6 \mathrm{mV}$ ohmic potential drop at the electrode surface. (b) Fluid streamlines (white) and flow speed (color scale). (c) Particle trajectories (white lines) and excess anion concentration (color scale, expressed as a ratio of the bulk salt concentration). Panels (a-c) correspond to $0.67 \mathrm{mM}$ mediator concentration and $5 \mathrm{mM}$ salt concentration $(\gamma=7.5)$. (d) Same as (c) for $0.15 \mathrm{mM}$ salt $(\gamma=0.22$ ).

an electric field (Figure 1a). An EOF is thus created that advects fluid along the negatively charged surface shrouding the electrode. To compensate this flow, fluid is drawn toward the electrode from above (Figure 1b). Negatively charged microparticles move by both migration and convection in this environment, resulting in trajectories that reflect both modes of transport (Figure 1c and $1 \mathrm{~d}$ ).

The role of convection is clearly visible in Figure 1c and 1d, which shows that trajectories approaching the edge of the electrode are deflected to the side. This occurs because the velocity of a particle near the surface, $u_{p, \|}$, is the sum of electrophoretic and convective components,

$$
u_{p, \|}=\frac{\varepsilon E_{\|}}{\mu}\left(\zeta_{p}-\zeta_{s}\right)
$$

where $\zeta_{p}$ is the $\zeta$-potential of the particle. When $\zeta_{s}$ is more negative than $\zeta_{p}$, convection dominates and particles escape along the surface.

The trajectories depend sensitively on the salt concentration. When $\gamma \gg 1$, the shape of the pathways is essentially independent of the ionic strength. When $\gamma \ll 1$, on the other hand, the concentrations of oxidized mediator and its counterion exceed the bulk salt concentration near the electrode (Figure 1d). This local enrichment diminishes the electrophoretic force acting on the colloidal particles compared to that which would be encountered otherwise. The EOF is also locally diminished, but this effect is less pronounced because the local fluid velocity also depends on the EOF far from the electrode due to fluid incompressibility. Convection thus dominates over migration at low $\gamma$, and more particles evade the electrode.
To experimentally challenge these predictions, we performed experiments using $1 \mu \mathrm{m}$ diameter negatively charged polystyrene particles $(\zeta$-potential $=-30 \mathrm{mV}$ ) and positively charged amidine-coated latex beads $(\zeta$-potential $=+32 \mathrm{mV})$. A $d=10 \mu \mathrm{m}$ diameter glass-encased Pt UME was inserted in a cell allowing optical access. The glass surrounding the UME had a negative $\zeta$-potential $(\approx-55 \mathrm{mV}$ at $\mathrm{pH} 5) .{ }^{18}$ As redox mediator and supporting electrolyte we employed $0.67 \mathrm{mM}$ $1,1^{\prime}$-ferrocenedimethanol and various concentrations of $\mathrm{KCl}$, respectively. In cases where we specify "no added salt", the conductivity of the solution was $2 \mu \mathrm{S} / \mathrm{cm}$, which corresponds to an ionic strength of $\sim 30 \mu \mathrm{M} .{ }^{19}$ Unless indicated otherwise, a constant potential of $+0.35 \mathrm{~V}$ was applied to the UME with respect to a Pt pseudoreference electrode, corresponding to a highly oxidizing overpotential (cyclic voltammogram in Figure S5). The current was monitored while simultaneously imaging the motion of the particles. Additional details on Materials and Methods are given in the Supporting Information.

Figure 2 shows typical amperometric responses (traces at other salt concentrations are shown in Figures S2 and S3). At high salt concentration ( $5 \mathrm{mM} \mathrm{KCl}$, Figure $2 \mathrm{a})$, we observed a conventional current blockade: single particles were drawn by electrophoresis and adsorbed to the electrode surface, interfering with transport of the redox mediator and leading to discrete steps in the current (some steps also result from rearrangement of particles ${ }^{15}$ ). At a lower salt concentration ( $0.15 \mathrm{mM}$, Figure $2 \mathrm{~b}$ ), however, the response became more complex. A new type of dip-like event appeared in which the current temporarily decreased before returning to its initial value. This suggests that some particles approached the surface of the electrode but then, counterintuitively, left again. ${ }^{13,14,20,21}$ Finally, positively charged particles (Figure 2c) exhibited no 


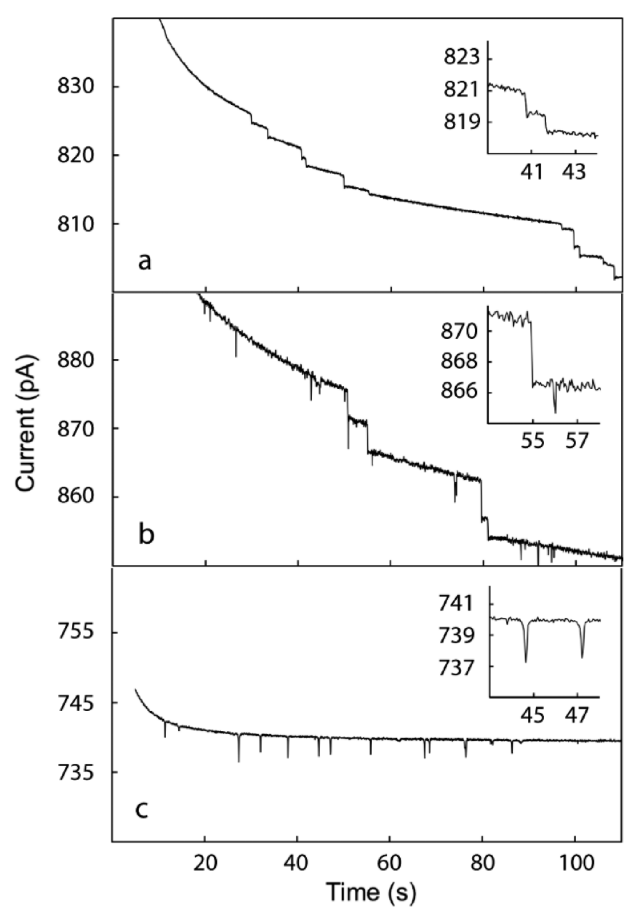

Figure 2. Current-time response for impact of negatively charged particles at high (5 mM, (a)) and low (0.15 mM, (b)) salt concentrations. While the response at high salt consists almost uniquely of stepwise decreases, dip-like features also occur at low salt. (c) Positively charged particles exhibit only dips $(\gamma=2)$.

steps but did show frequent dips. Steps would be unexpected in this case, since these particles migrate away from the electrode. The occurrence of dips indicates that the particles are transiently drawn to the electrode by a mechanism other than migration.

The origin of this bimodal behavior is readily elucidated by optically monitoring the particles (Supporting Information). Figure 3 shows snapshots of the trajectories of two particles at

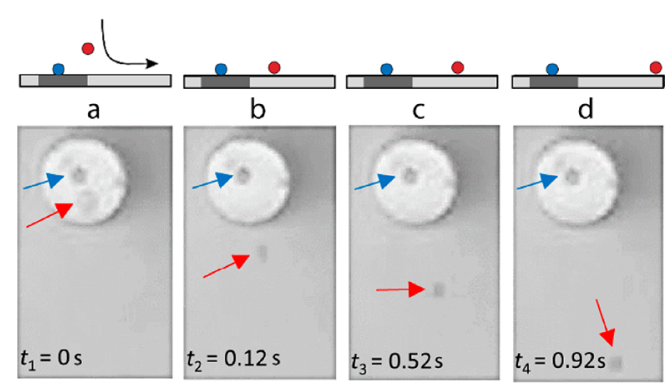

Figure 3. Trajectory for negatively charged particles. In (a), one microparticle is adsorbed to the electrode surface (blue arrow) while a second is out of focus in solution above the electrode (red arrow). The latter then approaches the surface and is deflected sideways ( $b-$ d). The diagrams above each image illustrate the different steps of this process. The UME diameter was $10 \mu \mathrm{m}$.

low salt concentration. The first (blue arrow) adsorbed on the surface of the electrode, corresponding to a stepwise current decrease. The second particle (red arrow) instead approached the electrode from above before being deflected along the surface of the glass. This behavior caused a dip-like transient amperometric response when the particle passed near the surface of the electrode (see Supporting Movie 1 for complete sequence). Positively charged particles also exhibit similar trajectories (Movie 2). In each case, particles moving away from the electrode stay close to the glass surface, matching the behavior shown in Figure 1d.

Figure 4 (orange curve) shows the radial distance from the center of the electrode versus time for a typical trajectory. The

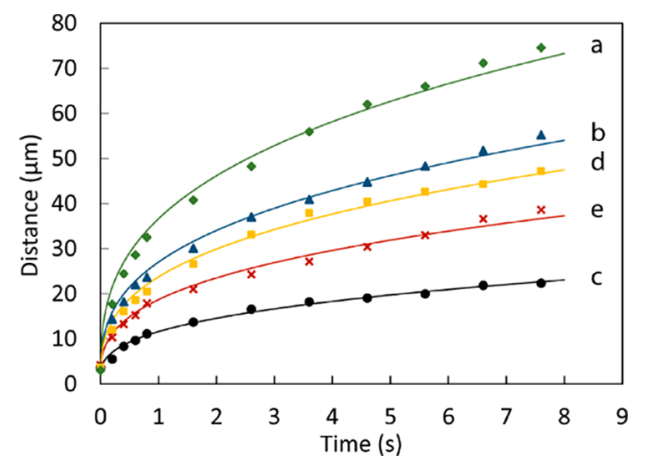

Figure 4. Distance-time curve for different particle charge, salt concentrations and applied potentials. $(\mathrm{a}-\mathrm{c})$ Positively charged particles with no added salt, $0.15 \mathrm{mM}$, and $1.34 \mathrm{mM}$ salt concentrations, respectively (applied potential: $0.35 \mathrm{~V}$ ). (d) Negatively charged particle at $0.15 \mathrm{mM}$ salt $(0.35 \mathrm{~V})$. (e) Positively charged particle with no added salt $(0.05 \mathrm{~V})$. The solid lines are fits to distance $\propto\left(t-t_{0}\right)^{1 / 3}$.

slope of this curve, which represents radial velocity, decreases as time progresses. This behavior is easily understood by noting that the magnitude of the electric field decreases as $r^{-2}$.,16 Combined with eq 1 , this leads to the prediction that the position along the surface obeys the form $r(t) \propto t^{1 / 3}$ (Supporting Information). Fits to this expression (solid lines in Figure 4) agree with the experimental data very well, showing that the particles move with velocity $u_{p, \|} \approx 50-100 \mu \mathrm{m} / \mathrm{s}$ in the vicinity of the electrode. This corresponds to Peclet number $\mathrm{Pe}=u_{p, \|} d / D \approx 10^{3}$, where $D$ is the diffusion coefficient of the particles, indicating that migration and convection dominate over diffusion in mass transport.

Equation 1 makes several testable predictions. First, positively charged particles $\left(\zeta_{p}>0\right)$ should move faster near the surface since migration and EOF work in concert. This is indeed observed experimentally (Figure 4, blue vs orange curve). Second, diminishing the strength of the electric field should lead to slower motion. The induced electric field is proportional to the magnitude of the faradaic current, which can be controlled via the applied electrode potential. We changed the electrode bias to $+0.05 \mathrm{~V}$, and as a result, the particles slowed down by a factor $\sim 2$ (Figure 4, red curve; Movie 3). We also changed the salt concentration, which resulted in higher particle velocities at lower salt (Figure 4, black, blue, and green curves; Movie 4 corresponds to the black curve). Third, making the surface potential $\zeta_{s}$ less negative or positive should diminish or even reverse the EOF. ${ }^{22}$ Measurements with a UME coated with the cationic polymer Poly-L-lysine exhibited once again step-like responses and the particle trajectories reversed direction, suggesting EOF suppression or reversal $\left(\zeta_{p}-\zeta_{s}<0\right.$ in eq 1; Figure S4, Movie 5).

Our results demonstrate that faradaic reactions induce convection in the fluid surrounding an electrode due to EOFs. This applies to both UMEs and electrodes imbedded in microsystems. It was previously inferred that convection 
influences measurements with UMEs at low salt concentrations. ${ }^{23}$ Our measurements fully support this conclusion and identify the intrinsic mechanism responsible for convection. Transport of the redox mediator is also affected, although the effect is less dramatic due to the relative importance of diffusion for small molecules $(\mathrm{Pe} \approx 1) .{ }^{16}$ Cyclic voltammetry indeed shows an increase on the order of $10 \%$ of the mediator mass-transport-limited current at low salt concentration (Figures S5 and S6). This is a priori surprising given that this molecule is electrically neutral and illustrates the generality of the EOF effect.

In addition, our results are relevant for the interpretation of impact electrochemistry measurements, which are often conducted under low salt conditions to increase the event frequency. EOFs generated by a mediator (for current blockade impact), electrocatalytic particles ${ }^{24,25}$ (for catalytic impact measurements), or even parasitic background reactions (for all impact methods) affect the rate and shape of events under these conditions, which must be taken into account for quantitative applications in single-entity electrochemistry. For example, our observations explain the previously reported saturation of the collision rate for $\gamma<1$. $^{26}$

On the other hand, a drawback of most impact methods is that electrodes become saturated over the course of an experiment, complicating interpretation and limiting measurement times. Inverting the charge of the particles instead leads to current dips without adsorption to the electrode surface, enabling extended measurements. The same could be achieved with negatively charged particles by using a reduction reaction for the mediator instead of an oxidation, thus reversing the polarity of the induced electric field.

\section{ASSOCIATED CONTENT}

\section{SI Supporting Information}

The Supporting Information is available free of charge at https://pubs.acs.org/doi/10.1021/jacs.0c08450.

Description of theoretical model, materials and methods, additional experiments (PDF)

COMSOL simulation report (ZIP)

Movie 1: Collision of negatively charged particles (MP4)

Movie 2: Collision of positively charged particles (MP4)

Movie 3: Switching electrode bias from +0.35 to $+0.05 \mathrm{~V}$ (MP4)

Movie 4: High salt concentration (MP4)

Movie 5: PLL-coated surface (MP4)

\section{AUTHOR INFORMATION}

\section{Corresponding Author}

Serge G. Lemay - MESA+ Institute for Nanotechnology, University of Twente, 7500 AE Enschede, The Netherlands; ○ orcid.org/0000-0002-0404-3169; Email: s.g.lemay@ utwente.nl

\section{Authors}

Taghi Moazzenzade - MESA+ Institute for Nanotechnology, University of Twente, 7500 AE Enschede, The Netherlands; (1) orcid.org/0000-0002-3908-6062

Xiaojun Yang - MESA+ Institute for Nanotechnology, University of Twente, 7500 AE Enschede, The Netherlands

Luc Walterbos - MESA+ Institute for Nanotechnology, University of Twente, 7500 AE Enschede, The Netherlands
Jurriaan Huskens - MESA+ Institute for Nanotechnology, University of Twente, 7500 AE Enschede, The Netherlands; (1) orcid.org/0000-0002-4596-9179

Christophe Renault - Physique de la Matiere Condensée, Ecole Polytechnique, CNRS, IP Paris 91128, Palaiseau, France; (1) orcid.org/0000-0003-4525-8702

Complete contact information is available at:

https://pubs.acs.org/10.1021/jacs.0c08450

\section{Notes}

The authors declare no competing financial interest.

\section{ACKNOWLEDGMENTS}

We acknowledge financial support from the TopSector HighTech Systems \& Materials in the TKI project "Early cancer diagnostics". C.R. further thanks the CNRS and the Agence Nationale de la Recherche (ANR-17-CE09-0034-01, "SEE") for funding.

\section{REFERENCES}

(1) Bard, A. J. F., Faulkner, L. R. Electrochemical Methods: Fundamentals and Applications, 2nd ed.; John Wiley \& Sons: New York, 2000; pp 137-157.

(2) Amatore, C.; Deakin, M. R.; Wightman, R. M. Electrochemical Kinetics at Microelectrodes: Part IV. Electrochemistry in Media of Low Ionic Strength. J. Electroanal. Chem. Interfacial Electrochem. 1987, 225 (1), 49-63.

(3) Oldham, K. B. Theory of Microelectrode Voltammetry with Little Electrolyte. J. Electroanal. Chem. Interfacial Electrochem. 1988, 250 (1), 1-21.

(4) Drew, S. M.; Wightman, R. M.; Amatore, C. A. Voltammetry of Ferrocene in Low Electrolyte Solutions. J. Electroanal. Chem. Interfacial Electrochem. 1991, 317 (1-2), 117-124.

(5) Bond, A. M.; Lay, P. A. Cyclic Voltammetry at Microelectrodes in the Absence of Added Electrolyte Using a Platinum Quasireference Electrode. J. Electroanal. Chem. Interfacial Electrochem. 1986, 199 (2), 285-295.

(6) Howell, J. O.; Wightman, R. M. Ultrafast Voltammetry and Voltammetry in Highly Resistive Solutions with Microvoltammetric Electrodes. Anal. Chem. 1984, 56 (3), 524-529.

(7) Amatore, C.; Knobloch, K.; Thouin, L. First Direct Experimental Evidence of Migration Contributions Through Monitoring of Concentration Profiles at Low Supporting Electrolyte Concentration. Electrochem. Commun. 2004, 6 (9), 887-891.

(8) Novev, J. K.; Compton, R. G. Natural Convection Effects in Electrochemical Systems. Curr. Opin. Electrochem. 2018, 7, 118-129.

(9) Paxton, W. F.; Kistler, K. C.; Olmeda, C. C.; Sen, A.; St. Angelo, S. K.; Cao, Y.; Mallouk, T. E.; Lammert, P. E.; Crespi, V. H. Catalytic Nanomotors: Autonomous Movement of Striped Nanorods. J. Am. Chem. Soc. 2004, 126 (41), 13424-13431.

(10) Kline, T. R.; Paxton, W. F.; Wang, Y.; Velegol, D.; Mallouk, T. E.; Sen, A. Catalytic micropumps: Microscopic Convective Fluid Flow and Pattern Formation. J. Am. Chem. Soc. 2005, 127 (49), 1715017151.

(11) Kostiuchenko, Z. A.; Glazer, P. J.; Mendes, E.; Lemay, S. G. Chemical Physics of Electroactive Materials - the Oft-overlooked Faces of Electrochemistry. Faraday Discuss. 2017, 199 (0), 9-28.

(12) Branagan, S. P.; Contento, N. M.; Bohn, P. W. Enhanced Mass Transport of Electroactive Species to Annular Nanoband Electrodes Embedded in Nanocapillary Array Membranes. J. Am. Chem. Soc. 2012, 134 (20), 8617-8624.

(13) Thorgaard, S. N.; Jenkins, S.; Tarach, A. R. Influence of Electroosmotic Flow on Stochastic Collisions at Ultramicroelectrodes. Anal. Chem. 2020, 92 (18), 12663-12669. 
(14) Quinn, B. M.; van't Hof, P. G.; Lemay, S. G. Time-resolved Electrochemical Detection of Discrete Adsorption Events. J. Am. Chem. Soc. 2004, 126 (27), 8360-8361.

(15) Boika, A.; Thorgaard, S. N.; Bard, A. J. Monitoring the Electrophoretic Migration and Adsorption of Single Insulating Nanoparticles at Ultramicroelectrodes. J. Phys. Chem. B 2013, 117 (16), 4371-4380.

(16) Moazzenzade, T.; Huskens, J.; Lemay, S. G. Stochastic Electrochemistry at Ultralow Concentrations: the Case for Digital Sensors. Analyst 2020, 145 (3), 750-758.

(17) Probstein, R. F. Physicochemical Hydrodynamics: An Introduction, 2nd ed.; John Wiley \& Sons: 1994; pp 165-210.

(18) Gu, Y.; Li, D. The $\zeta$-Potential of Glass Surface in Contact with Aqueous Solutions. J. Colloid Interface Sci. 2000, 226 (2), 328-339.

(19) Marion, G. M.; Babcock, K. L. Predicting Specific Conductance and Salt Concentration in Dilute Aqueous Solutions. Soil Sci. 1976, 122 (4), 181-187.

(20) Ronspees, A. T.; Thorgaard, S. N. Blocking Electrochemical Collisions of Single E. coli and B. subtilis Bacteria at Ultramicroelectrodes Elucidated Using Simultaneous Fluorescence Microscopy. Electrochim. Acta 2018, 278, 412-420.

(21) Yoo, J. J.; Anderson, M. J.; Alligrant, T. M.; Crooks, R. M. Electrochemical Detection of Insulating Beads at Subattomolar Concentration via Magnetic Enrichment in a Microfluidic Device. Anal. Chem. 2014, 86 (9), 4302-4307.

(22) Subramanian, S.; Catchmark, J. M. Control of Catalytically Generated Electroosmotic Fluid Flow through Surface Zeta Potential Engineering. J. Phys. Chem. C 2007, 111 (32), 11959-11964.

(23) Bento, M. F.; Thouin, L.; Amatore, C. Potential Measurements in Steady State Voltammetry at Low Electrolyte/Analyte Concentration Ratios. Role of Convection on Ohmic Drop: A Simplified Model. J. Electroanal. Chem. 1998, 446 (1), 91-105.

(24) Xiao, X.; Bard, A. J. Observing Single Nanoparticle Collisions at an Ultramicroelectrode by Electrocatalytic Amplification. J. Am. Chem. Soc. 2007, 129 (31), 9610-9612.

(25) Xiao, X.; Fan, F.-R. F.; Zhou, J.; Bard, A. J. Current Transients in Single Nanoparticle Collision Events. J. Am. Chem. Soc. 2008, 130 (49), 16669-16677.

(26) Renault, C.; Lemay, S. G. Electrochemical Collisions of Individual Graphene Oxide Sheets: An Analytical and Fundamental Study. ChemElectroChem 2020, 7 (1), 69-73. 